\title{
Astroglial Glutamate-Glutamine Shuttle Is Involved in Central Sensitization of Nociceptive Neurons in Rat Medullary Dorsal Horn
}

\author{
Chen-Yu Chiang, ${ }^{1 \star}$ Jing Wang, ${ }^{1 \star}$ Yu-Feng Xie, ${ }^{1}$ Sun Zhang, ${ }^{1}$ James W. Hu, ${ }^{1}$ Jonathan O. Dostrovsky, ${ }^{2}$ and \\ Barry J. Sessle 1,2 \\ ${ }^{1}$ Faculty of Dentistry, University of Toronto, Toronto, Ontario, Canada M5G 1G6, and 2Department of Physiology, Faculty of Medicine, University of \\ Toronto, Toronto, Ontario, Canada M5S 1A8
}

\begin{abstract}
Growing evidence suggests that astroglia are involved in pain states, but no studies have tested their possible involvement in modulating the activity of nociceptive neurons per se. This study has demonstrated that the central sensitization induced in functionally identified nociceptive neurons in trigeminal subnucleus caudalis (the medullary dorsal horn) by application of an inflammatory irritant to the rat's tooth pulp can be significantly attenuated by continuous intrathecal superfusion of methionine sulfoximine (MSO; $0.1 \mathrm{mM})$, an inhibitor of the astroglial enzyme glutamine synthetase that is involved in the glutamate-glutamine shuttle. Simultaneous superfusion of MSO and glutamine $(0.25 \mathrm{~mm})$ restored the irritant-induced central sensitization. In control experiments, superfusion of either MSO or glutamine alone, or vehicle, did not produce any significant changes in neuronal properties. These findings suggest that the astroglial glutamateglutamine shuttle is essential for the initiation of inflammation-induced central sensitization but that inhibition of astroglial function may not affect normal nociceptive processing.
\end{abstract}

Key words: glutamine synthetase; methionine sulfoximine; trigeminal subnucleus caudalis; mustard oil; tooth pulp; inflammatory pain

\section{Introduction}

There is evidence that glia-neuron interactions are involved in mechanisms underlying the development of chronic pain (Watkins et al., 2001; Miller, 2005; Raivich, 2005; Watkins and Maier, 2005; Araque, 2006; Woolf and Salter, 2006). These studies have especially focused on activation of microglia (Tsuda et al., 2003, 2005; Inoue et al., 2004; Allen and Barres, 2005; Frank et al., 2007; Zhang et al., 2007), but there is growing evidence that astroglia may also be important (Watkins et al., 1997, 2001; Sweitzer et al., 1999; Raghavendra et al., 2004; Tanga et al., 2004; Haydon and Carmignoto, 2006; Qin et al., 2006; Lan et al., 2007). Central sensitization is considered a crucial process underlying the development of chronic pain states (Dubner and Basbaum, 1994; Sessle, 2000; Woolf and Salter, 2006), and we have recently demonstrated that intrathecal application of fluoroacetate (FA), a specific inhibitor of the metabolic enzyme aconitase, which is a component of the tricarboxylic acid cycle in astroglia (Fonnum et al., 1997; Schousboe and Waagepetersen, 2006), attenuates cen-

Received May 17, 2007; revised June 25, 2007; accepted July 7, 2007.

This work was supported by National Institutes of Health Grant DE-04786 (B.J.S.) and Canadian Institutes of Health Research Grant MOP-82831 (J.0.D.). We gratefully acknowledge Dr. J.-C. Lee for his graphical assistance and K. Macleod and S. Carter for their technical assistance.

${ }^{*}$ C.-Y.C. and J.W. contributed equally to this work.

Correspondence should be addressed to Dr. Barry J. Sessle, Faculty of Dentistry, University of Toronto, 124 Edward Street, Toronto, Ontario, Canada M5G 1G6. E-mail: barry.sessle@utoronto.ca.

J. Wang's present address: Department of Orthopaedics Research Institute, The Second Hospital, Lanzhou University, Lanzhou, People's Republic of China.

DOI:10.1523/JNEUROSCI.2260-07.2007

Copyright $\odot 2007$ Society for Neuroscience $\quad$ 0270-6474/07/279068-09\$15.00/0 tral sensitization in functionally identified nociceptive neurons of trigeminal subnucleus caudalis without affecting normal nociceptive processing (Xie et al., 2007). Our findings are consistent with the literature that glia may not play a significant role in normal nociceptive processing but rather are contributing to pathological pain states (Raghavendra et al., 2004; Watkins and Maier, 2005).

Glutamate plays a major role in central sensitization (Dubner and Basbaum, 1994; Sessle, 2000; Woolf and Salter, 2006), and the glutamate-glutamine shuttle is a key process of astroglial function. This shuttle includes the uptake of excessive extrasynaptic glutamate and the production [via glutamine synthetase (GS)] and release from astroglia of glutamine, which is then taken up by neuronal elements to replenish the supply of glutamate (Zwingmann and Leibfritz, 2003; Hertz, 2004; Hertz and Zielke, 2004; Fonseca et al., 2005). A potent inhibitor of GS in astroglia is methionine sulfoximine (MSO), and its effects can be counteracted by glutamine application (Bacci et al., 2002; Blin et al., 2002; Suarez et al., 2002; Shin et al., 2003; Gibbs and Hertz, 2005; Tanigami et al., 2005; Liang et al., 2006). However, only one study has used MSO to investigate the involvement of astroglial GS in the effects of noxious stimuli on glutamatergic receptor-related events in astroglia (Muscoli et al., 2005), and no studies have tested its effects on central sensitization in functionally identified nociceptive neurons. Therefore, the aim of the present study was to test whether intrathecal superfusion of MSO can attenuate central sensitization induced in functionally identified caudalis nociceptive neurons by application of an inflammatory irritant to 
the rat's tooth pulp, and whether intrathecal superfusion of glutamine can restore the central sensitization. Data have been partly reported in abstract form (Chiang et al., 2006).

\section{Materials and Methods}

Animals. The experiments were performed in 44 anesthetized rats. The methods used for animal preparation, stimulation, and neuronal recording and classification were similar to those described previously in detail (Chiang et al., 1998, 2005; Xie et al., 2007) and so will only be briefly outlined here. Male Sprague Dawley adult rats $(280-400 \mathrm{~g})$ were anesthetized by a single intraperitoneal injection of a mixture of $\alpha$-chloralose $(50 \mathrm{mg} / \mathrm{kg})$ and urethane $(1 \mathrm{~g} / \mathrm{kg})$. Then a tracheal cannula was inserted, and the left external jugular vein was cannulated. To expose the pulp of the right maxillary first molar, an occlusal cavity $(\sim 0.7 \mathrm{~mm}$ in diameter and $0.6-0.8 \mathrm{~mm}$ in depth) was performed with a dental drill (Rotrex 780, at a moderate speed) carrying a Carbide bur $(0.5 \mathrm{~mm}$ in diameter). During drilling, the cleaned molar occlusal surface was cooled 3-5 times by the cotton pellet soaked with cooled saline. Then, the cavity was immediately filled with a small piece of cotton pellet soaked with normal saline. After the rat was placed in a stereotaxic apparatus, the caudal medulla was surgically exposed, and the overlying dura and subarachnoid membrane were removed. Just before the neuronal recording session, a supplemental dose of urethane (200-300 mg/kg, i.v.) was administered, and the rat was then immobilized with intravenous pancuronium bromide [initial dose, $0.3-0.4 \mathrm{ml}$ of $1 \mathrm{mg} / \mathrm{ml}$ solution, followed by a continuous intravenous infusion of a mixture of $70 \%$ urethane solution $(0.2 \mathrm{~g} / \mathrm{ml})$ and $30 \%$ pancuronium solution $(1 \mathrm{mg} / \mathrm{ml})$ at a rate of $0.4-0.5$ $\mathrm{ml} / \mathrm{h}$ ] and artificially ventilated throughout the whole experimental period. A deep level of anesthesia was confirmed periodically by the lack of spontaneous movements and responses to pinching the paw when pancuronium-induced muscle paralysis was allowed to wear off. Heart rate, percentage expired $\mathrm{CO}_{2}$, and rectal temperature were constantly monitored and maintained at physiological levels of 333-430 beats/min, $3.5-4.2 \%$, and $37-37.5^{\circ} \mathrm{C}$, respectively. All surgeries and procedures were approved by the University of Toronto Animal Care Committee in accordance with the regulations of the Ontario Animal Research Act (Canada).

Recording and stimulation procedures. Single neuronal activity was recorded extracellularly by means of an epoxy resin-coated tungsten microelectrode. As the microelectrode was advanced with a rostral inclination of $23^{\circ}$ into the right caudal medulla, $1.4-2.0 \mathrm{~mm}$ lateral to the midline and 1.5-2.0 mm behind the obex, stimuli (see below) were applied to the orofacial tissues to search for caudalis nociceptive neurons receiving an orofacial sensory input. Neuronal activity was amplified, displayed on oscilloscopes, and led to a window discriminator and an analog-to-digital converter (CED 1401 plus; Cambridge Electronic Design, Cambridge, UK) connected to a personal computer running Spike2 software (Cambridge Electronic Design), which digitized and stored the triggered action potentials. Data were analyzed off-line with this same software.

A wide range of mechanical (brush, pressure, and pinch), and noxious thermal (radiant heat; $51-53^{\circ} \mathrm{C}$ ) stimuli were applied to the orofacial region to classify each neuron as low-threshold mechanoreceptive, wide dynamic range (WDR), or nociceptive specific (NS) (Chiang et al., 1998, 2005; Xie et al., 2007). Only WDR and NS neurons were further tested in this study. The average spontaneous activity in hertz of a nociceptive neuron was determined over the initial $1 \mathrm{~min}$ (for NS neurons) or $5 \mathrm{~min}$ (for WDR neurons) recording period. As outlined in our previous studies (Chiang et al., 1998, 2005; Park et al., 2001; Hu et al., 2002; Xie et al., 2007), the cutaneous mechanoreceptive field (RF) of each WDR neuron was determined by brushing the skin with a force $<2 \mathrm{~g}$; the cutaneous pinch/pressure RF of each NS or WDR neuron was determined through the use of a blunt probe and a pair of nonserrated forceps. A burst response consisting of at least two spikes during each stimulus trial was accepted as the criterion for the RF boundary of the neuron tested. Noxious cutaneous stimulation was used sparingly so as to avoid damage to the skin and the production of peripheral sensitization. A deep nociceptive input was considered to occur only if application of the blunt probe

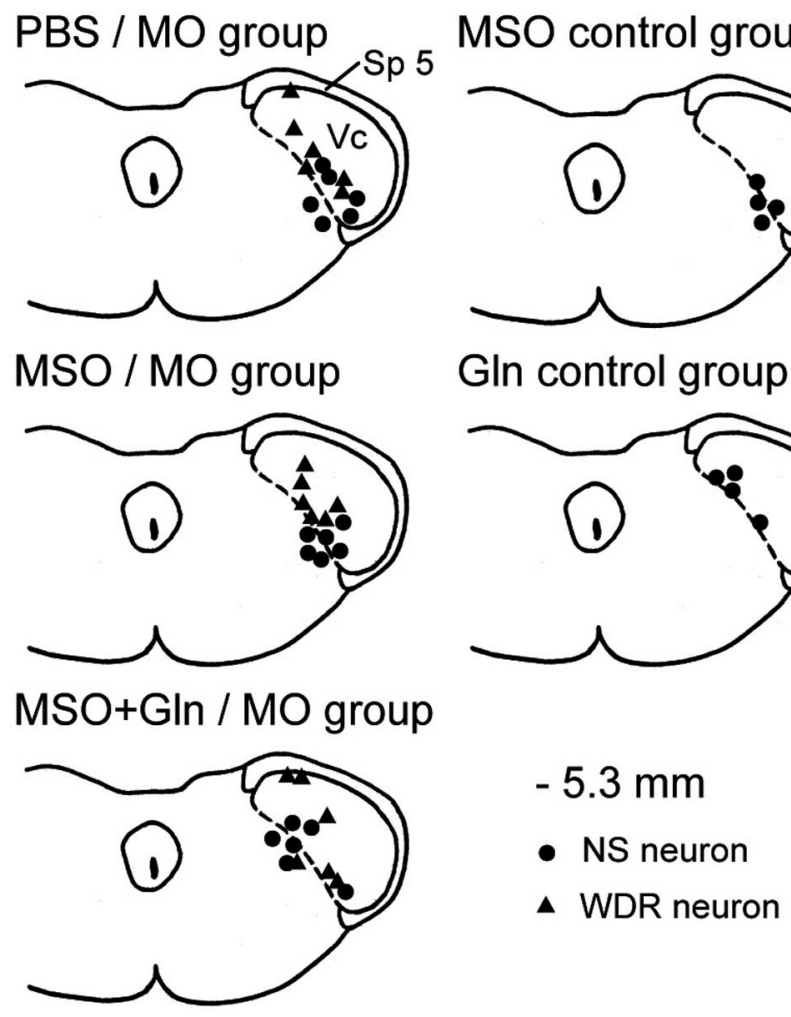

Figure 1. Histologically confirmed neuronal recording sites in the MDH. The recording sites of neurons in each of the groups were plotted separately onto a section of the caudalis medulla at $5.3 \mathrm{~mm}$ behind interaural line. Note that the sites of all NS neurons and the majority of WDR neurons were located in the deep laminas of trigeminal subnucleus caudalis; the remaining three WDR neurons were located in the superficial laminas. Vc, Trigeminal subnucleus caudalis, also termed MDH; Sp 5, trigeminal spinal tract.

to the skin overlying muscle or bone evoked a neuronal response at a mechanical threshold $>5 \mathrm{~g}$, but no response could be evoked by the wide range of mechanical pinch or thermal stimuli applied to the skin itself (Yu et al., 1993; Chiang et al., 1998, 2005). For WDR neurons, the activation threshold was defined as the von Frey nylon monofilament with the lowest value $(0.012 \mathrm{~g})$ that elicited 1 or 2 spikes per trial in at least 5 of 6 trials. For NS neurons, the activation threshold to a mechanical stimulus applied to the orofacial RF was assessed by a pair of forcemonitoring forceps (with an attached strain gauge that monitored force levels up to $600 \mathrm{~g} / \mathrm{mm}^{2}$ ) or an electronic von Frey monofilament, and as the mechanical force was gradually increased, the responses of the tested neuron were monitored and recorded simultaneously by the use of the Spike2 program (CED 1401 plus). The NS neuronal responses to four incremental graded mechanical pinch stimuli with a force-monitoring forceps or graded pressure by means of electronic von Frey monofilament applications (e.g., 20-25, 40-50, 60-80, 100, and even $200 \mathrm{~g}$, delivered in ascending order, each for $5 \mathrm{~s}$ at an interval of $>45 \mathrm{~s}$ ) applied to the neuronal orofacial RF were determined, as described previously (Chiang et al., 1998, 2005; Park et al., 2001; Hu et al., 2002; Xie et al., 2007). The pinch/pressure-evoked responses of a given neuron were assessed by summing the number of spikes evoked by each of these four graded stimuli.

Chemicals. The chemicals used included allyl isothiocyanate (MO; 95\%; Sigma-Aldrich, St. Louis, MO); L-MSO (0.1 mm; Sigma-Aldrich); L-glutamine (0.25 mM; Sigma-Aldrich); PBS, pH 7.4, as vehicle (SigmaAldrich). All chemicals except MO were freshly dissolved in PBS.

Experimental paradigm. As described previously (Chiang et al., 1998, 2005; Xie et al., 2007), MO was applied to the tooth pulp to evoke caudalis central sensitization, which was reflected in an increase in spontaneous activity, RF size, responses to noxious stimuli, and a decrease in mechanical activation threshold. Three groups (PBS/MO, MSO/MO, and 
Table 1. Effects of intrathecal superfusion of MSO, MSO plus glutamine, or PBS on features of M0/pulp-induced central sensitization in MDH nociceptive neurons

\begin{tabular}{|c|c|c|c|c|}
\hline Treatment & Tactile RF $\left(\mathrm{cm}^{2}\right)$ & Pinch or pressure $\mathrm{RF}\left(\mathrm{cm}^{2}\right)$ & Mechanical activation threshold (g) & $\begin{array}{l}\text { Responses to graded } \\
\text { stimuli (sum of spikes) }\end{array}$ \\
\hline \multicolumn{5}{|l|}{ PBS/M0 experiments } \\
\hline \multicolumn{5}{|l|}{ NS neurons $(n=6)$} \\
\hline Baseline value & 0 & $1.9 \pm 0.6$ & $99.3 \pm 38.6$ & $36.1 \pm 5.8$ \\
\hline Value 18 min after PBS & 0 & $1.9 \pm 0.5$ & $97.1 \pm 35.5$ & $35.7 \pm 9.7$ \\
\hline Value 18 min after M0 & $0.06 \pm 0.03^{*}$ & $4.9 \pm 0.9^{*}$ & $49.5 \pm 16.5^{*}$ & $129.5 \pm 40.8^{*}$ \\
\hline Value $48 \mathrm{~min}$ after $\mathrm{M} 0$ & 0 & $4.2 \pm 0.8^{*}$ & $83.8 \pm 30.4$ & $87.3 \pm 45.8$ \\
\hline \multicolumn{5}{|l|}{ WDR neurons $(n=6)$} \\
\hline Baseline value & $0.76 \pm 0.39$ & $2.4 \pm 0.9$ & $1.01 \pm 0.95$ & \\
\hline Value 18 min after PBS & $0.84 \pm 0.45$ & $2.5 \pm 0.9$ & $1.01 \pm 0.95$ & \\
\hline Value 18 min after M0 & $1.03 \pm 0.35^{*}$ & $4.1 \pm 1.5^{*}$ & $0.07 \pm 0.05^{*}$ & \\
\hline Value $48 \mathrm{~min}$ after $\mathrm{MO}$ & $0.79 \pm 0.36$ & $2.6 \pm 0.8$ & $1.23 \pm 1.15$ & \\
\hline \multicolumn{5}{|l|}{ MSO/M0 experiments } \\
\hline \multicolumn{5}{|l|}{ NS neurons $(n=6)$} \\
\hline Baseline value & 0 & $2.8 \pm 0.4$ & $116.5 \pm 36.4$ & $89.0 \pm 28.7$ \\
\hline Value 18 min after MSO & 0 & $3.0 \pm 0.3$ & $94.5 \pm 25.5$ & $63.8 \pm 11.6$ \\
\hline Value 18 min after M0 & 0 & $2.9 \pm 0.3$ & $112.5 \pm 28.4$ & $53.8 \pm 15.6$ \\
\hline Value $48 \mathrm{~min}$ after $\mathrm{M} 0$ & 0 & $2.6 \pm 0.4$ & $113.5 \pm 29.7$ & $44.8 \pm 13.2$ \\
\hline \multicolumn{5}{|l|}{ WDR neurons $(n=6)$} \\
\hline Baseline value & $0.81 \pm 0.12$ & $3.6 \pm 0.8$ & $0.44 \pm 0.37$ & \\
\hline Value $18 \mathrm{~min}$ after MSO & $0.78 \pm 0.19$ & $3.4 \pm 0.8$ & $0.44 \pm 0.37$ & \\
\hline Value 18 min after M0 & $0.68 \pm 0.23$ & $3.4 \pm 0.7$ & $0.33 \pm 0.23$ & \\
\hline Value $48 \mathrm{~min}$ after $\mathrm{M} 0$ & $0.52 \pm 0.16$ & $3.1 \pm 0.7$ & $0.44 \pm 0.37$ & \\
\hline \multicolumn{5}{|l|}{$\mathrm{MSO}+\mathrm{G} \ln / \mathrm{MO}$ experiments } \\
\hline \multicolumn{5}{|l|}{ NS neurons $(n=6)$} \\
\hline Baseline value & 0 & $3.0 \pm 0.8$ & $101.7 \pm 23.7$ & $122.7 \pm 28.7$ \\
\hline Value 18 min after MSO & $0.02 \pm 0.02$ & $3.4 \pm 0.9$ & $99.0 \pm 30.9$ & $128.7 \pm 29.5$ \\
\hline \multicolumn{5}{|l|}{$+G \ln$} \\
\hline Value 18 min after M0 & $0.43 \pm 0.27^{*}$ & $4.9 \pm 1.1^{*}$ & $73.5 \pm 21.7^{*}$ & $177.3 \pm 26.5$ \\
\hline Value $48 \mathrm{~min}$ after M0 & $0.16 \pm 0.16$ & $3.8 \pm 0.8^{*}$ & $99.5 \pm 21.6$ & $146.3 \pm 37.1$ \\
\hline \multicolumn{5}{|l|}{ WDR neurons $(n=6)$} \\
\hline Baseline value & $0.71 \pm 0.23$ & $3.6 \pm 1.1$ & $0.06 \pm 0.03$ & \\
\hline Value 18 min after MSO & $0.74 \pm 0.18$ & $3.5 \pm 0.8$ & $0.06 \pm 0.03$ & \\
\hline \multicolumn{5}{|l|}{$+G \ln$} \\
\hline Value $18 \mathrm{~min}$ after M0 & $0.88 \pm 0.33$ & $6.2 \pm 1.5^{*}$ & $0.02 \pm 0.01^{*}$ & \\
\hline Value $48 \mathrm{~min}$ after M0 & $0.84 \pm 0.31$ & $3.9 \pm 1.1$ & $0.08 \pm 0.02$ & \\
\hline \multicolumn{5}{|l|}{ Statistical comparisons } \\
\hline \multicolumn{5}{|l|}{ NS neurons } \\
\hline MSO/MO vs PBS/M0 ${ }^{a}$ & ns & $F_{(1,80)}=61.79 ; p<0.001$ & $F_{(1,80)}=16.9 ; p<0.001$ & $F_{(1,80)}=19.2 ; p<0.001$ \\
\hline MSO/MO vs MSO+Gln/ & $F_{(1,80)}=9.26 ; p<0.01$ & $F_{(1,80)}^{(1,00)}=21.90 ; p<0.001$ & $F_{(1,80)}=12.9 ; p<0.001$ & $F_{(1,80)}^{11,00)}=5.53 ; p<0.02$ \\
\hline \multicolumn{5}{|l|}{$\mathrm{MO}^{a}$} \\
\hline \multicolumn{5}{|l|}{ WDR neurons } \\
\hline $\mathrm{MSO} / \mathrm{MO}$ vs $\mathrm{PBS} / \mathrm{MO}^{a}$ & $F_{(1,80)}=7.21 ; p<0.01$ & $F_{(1,80)}=10.34 ; p=0.002$ & $F_{(1,40)}=4.41 ; p<0.05$ & \\
\hline $\begin{array}{l}\mathrm{MSO} / \mathrm{MO} \text { vs } \mathrm{MSO}+\mathrm{Gln} / \\
\mathrm{M}^{a}\end{array}$ & $F_{(1,80)}=8.05 ; p<0.01$ & $F_{(1,80)}=14.98 ; p<0.001$ & $F_{(1,40)}=4.41 ; p<0.05$ & \\
\hline
\end{tabular}

All values are shown as mean \pm SEM. ns, Not significant. ${ }^{*} p<0.05$ for comparison between the baseline value and values at the different time after chemical or PBS in each group (RM ANOVA or RM ANOVA on ranks, followed by Dunnett's test).

${ }^{a}$ Two-way ANOVA results for comparison of all time points between MSO/MO and PBS/MO or MSO $+\mathrm{Gln} / \mathrm{MO}$ groups.

$\mathrm{MSO}+\mathrm{Gln} / \mathrm{MO}$ ) of animals were studied, and a standard assessment of neuronal spontaneous activity, mechanical activation threshold, orofacial RF size, and pinch/pressure-evoked responses to the four graded stimuli was performed periodically throughout the experiment. After baseline values of neuronal properties were obtained, MSO (MSO/MO group), MSO plus glutamine (MSO+Gln/MO group), or PBS (PBS/MO group, as a control) was continuously superfused (i.t.) over the ipsilateral medullary dorsal horn $(\mathrm{MDH}$; at a rate of $0.6 \mathrm{ml} / \mathrm{h})$. Five and fifteen minutes after the commencement of the superfusion, two assessments of neuronal properties were performed. Then, the saline-soaked cotton pellet was carefully removed from the molar pulp cavity and replaced with a segment of dental paper point soaked with MO $(0.2 \mu \mathrm{l})$. The cavity was promptly sealed with CAVIT (ESPE, Seefeld, Germany) to prevent MO from leaking out of the tooth and to ensure the chemical's sustained action on pulp afferents. Three minutes after MO application, the standard assessment of neuronal properties was repeatedly performed at 10 min intervals until $50 \mathrm{~min}$, except for the threshold assessment of WDR neurons that was performed at baseline, $15 \mathrm{~min}$ after MSO, glutamine, or PBS superfusion, and 18 and $48 \mathrm{~min}$ after MO application. The chemical intrathecal superfusion was terminated at the end of the experiment. Because of its specific effect on GS, MSO is a toxic agent disturbing astroglial function (Shaw and Bains, 1998; Shaw et al., 1999). In pilot experiments, we found that superfusion of several doses (from 4 to 0.25 $\mathrm{mM}$ ) of MSO produced strong depression of neuronal function, and only the $0.1 \mathrm{~mm}$ dose appeared to be appropriate for long-term superfusion over MDH without disturbing neuronal function (RF size, threshold, and response) but still being able to attenuate the MO-induced central sensitization. Thus, the $0.1 \mathrm{~mm}$ dose of MSO was used in this study.

In addition to these groups of animals, some other animals were used in which the effects of continuous intrathecal superfusion of MSO alone, glutamine alone, or glutamine followed by $\mathrm{MO}$ were tested. A protocol similar to that in the above-mentioned experiments was used, except that 


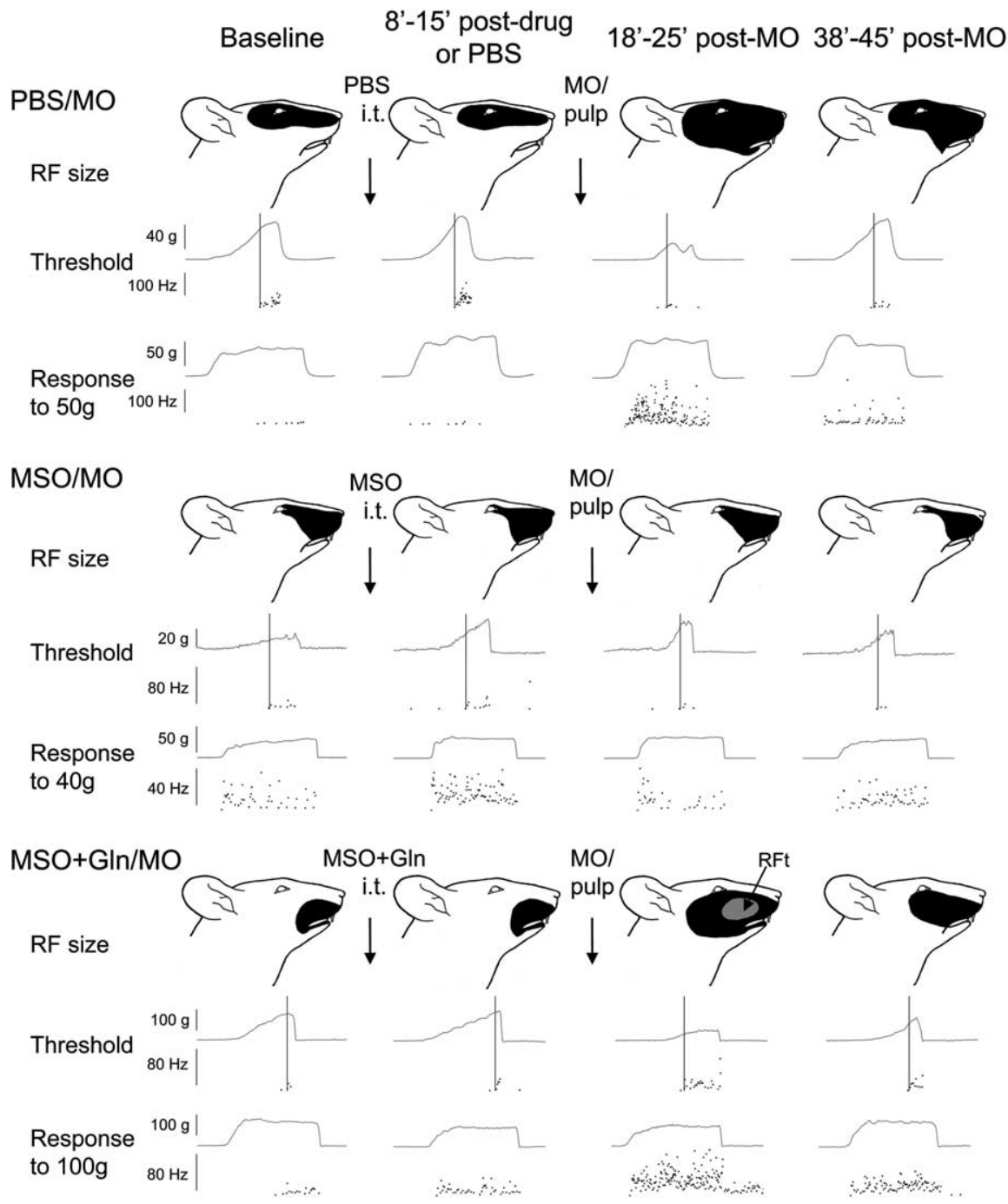

Figure 2. Examples showing MO-induced neuroplastic changes (central sensitization) in NS neurons after continuous intrathecal superfusion of PBS, MSO, or MSO plus glutamine over MDH. For each example, the pinch RF (top), activation threshold (middle), and responses to pinch/pressure stimuli (bottom) are shown. Data at baseline, 8-15 min after chemical or PBS superfusion (i.e., before $\mathrm{MO}$ application), and $18-25$ and $38-45 \mathrm{~min}$ after $\mathrm{MO}$ application of each NS neuron are arranged in columns from left to right. Note that the NS neurons illustrated from both the PBS/M0 and MSO + GIn/M0 groups showed M0-induced neuroplastic changes, whereas no such marked changes occurred in the NS neuron of the MSO group, indicating that simultaneous superfusion of glutamine could restore the MO-induced neuroplastic changes in NS neurons. The cutoff (vertical) line shows the activation threshold of a given neuron, and the neuronal discharges are displayed in an interspike instantaneous frequency distribution. The unit responses to pinch/pressure stimuli (1-2 times threshold intensity) are also shown in the instantaneous frequency distribution during the $5 \mathrm{~s}$ stimulation period. The black area indicates the pinch/pressure RF. RFt refers to that part of the RF from which the neuron could be activated by tactile as well as pinch/pressure stimulation.

Mann-Whitney $U$ test, respectively. The level of significance was set at $p<0.05$.

\section{Results}

Forty-four functionally identified NS $(n=$ $26)$ and WDR $(n=18)$ neurons responding to ipsilateral orofacial noxious stimulation were studied. The majority of these neurons were located in the deep laminas of caudalis, and their recording sites are shown in Figure 1. With the exception of the eight NS neurons that were tested as controls with MSO alone or glutamine alone, comparisons of neuronal properties at baseline were made between the NS $(n=18)$ and WDR $(n=15)$ neurons located in the deep laminas of caudalis: WDR neurons had a characteristic tactile RF with a very low mechanical activation threshold and were significantly different from the NS neurons in incidence of spontaneous activity ( 2 of 18 NS neurons vs 15 of 15 WDR neurons, respectively; $p<$ 0.001 , Fisher's test) and basal spontaneously firing rate $(0.08$ vs $2.1 \mathrm{~Hz}$, respectively; $p<0.001$, Mann-Whitney $U$ test) but not in pinch/pressure RF size $(p>$ $0.3)$. However, after $\mathrm{MO}$ application in the PBS/MO group (see below), the peak increase in pinch/pressure RF size between the NS neurons $(292 \pm 51 \% ; n=6)$ and WDR neurons $(197 \pm 39 \% ; n=6)$ was significantly different $(p<0.05$; MannWhitney $U$ test).

\section{Orofacial RF size}

In the PBS/MO group, all six NS neurons had at baseline an ipsilateral pinch/pressure RF; most neurons had a dumbbelllike shaped RF in tissues supplied by the maxillary division of the trigeminal nerve that involved the periorbital region, nose, and vibrissal pad. MO application to the pulp produced a significant, long-lasting increase in RF size in all six neurons that peaked at 18-28 min and that expanded into the trigeminal nerve's mandibular division in three neurons $(p<0.05)$ (Table 1, Figs. 2, 4A). In addition, soon after $\mathrm{MO}$ application, a novel tactile RF appeared for

no MO was applied to the pulp after the chemical intrathecal superfusion started. The standard assessment of neuronal properties was repeatedly performed at $10 \mathrm{~min}$ intervals throughout a $50 \mathrm{~min}$ observation period.

Histological and statistical analyses. Recording sites were marked by electrolytic lesions (anodal current of $8 \mu \mathrm{A}$ for $13 \mathrm{~s}$ ) and verified histologically (Fig. 1). Data are reported as mean \pm SEM. Statistical analyses were based on the normalized data (in percentage). Differences between the baseline value and values at different postchemical (i.e., MO, MSO, $\mathrm{PBS}$, and MSO plus glutamine) time points in each group were treated by repeated-measures ANOVA (RM ANOVA) or RM ANOVA on ranks, followed by Dunnett's test. Differences between the PBS/MO or $\mathrm{MSO}+\mathrm{Gln} / \mathrm{MO}$ group and the $\mathrm{MSO} / \mathrm{MO}$ group were treated by two-way ANOVA followed by Bonferroni $t$ test. The difference in incidence and firing rate of spontaneous activity, in RF size, and in responses to graded stimuli between NS and WDR neurons was treated by Fisher's test and
$10-20 \mathrm{~min}$ in three of the six neurons $(p<0.05)$ (Fig. $4 B$ ). Five of the six WDR neurons had at baseline an extensive ipsilateral orofacial pinch/pressure RF involving the periorbital and perioral regions; the remaining one neuron located in the superficial laminas had an RF limited to the vibrissal pad. MO application produced a significant increase in RF size in all six neurons $(p<$ 0.05 ) (Table 1, Figs. 3, 4A). The tactile RF of all six WDR neurons was located within the boundary of the pinch/pressure RF; only four of these neurons showed an increase in the tactile RF after MO application $(p<0.05)$ (Table 1, Figs. 3, $4 B$ ).

In the $\mathrm{MSO} / \mathrm{MO}$ group, all six NS neurons had at baseline an ipsilateral pinch/pressure RF involving the ophthalmic and/or maxillary divisions of the trigeminal nerve (Fig. 2) that, except for 
one neuron, remained unchanged after MSO superfusion and MO application ( $p>0.05)$; no novel tactile RF was noted in this group. The pinch/pressure RF and the tactile RF of the six WDR neurons involved mainly the maxillary and mandibular divisions of the trigeminal nerve (Fig. 3 ) and remained unchanged after application of both MSO and MO $(p>0.1)$. Overall, MSO pretreatment attenuated the MO-induced increase in RF size in both NS and WDR neurons $(p>0.2-0.7)$ (Figs. 2-4), and these effects were significantly different from those in the PBS/MO group $(p<0.01-0.001)$ (Table 1$)$.

In the $\mathrm{MSO}+\mathrm{Gln} / \mathrm{MO}$ group, the six NS neurons had at baseline an ipsilateral pinch/pressure RF involving mainly the trigeminal maxillary division. Although $\mathrm{MSO}+$ glutamine superfusion did not affect their RF size, MO application produced a long-lasting significant increase in RF size $(p<0.05)$ (Fig. 2, Table 1$)$. A novel tactile RF also appeared in five of the six neurons for 10-30 min after MO application $(p<0.05)$ (Table 1, Figs. 2, $4 B$ ). The pinch/pressure and tactile RF of the six WDR neurons involved mainly the trigeminal maxillary division (Fig. 3). Whereas MSO+glutamine superfusion produced a slight increase in both pinch/ pressure and tactile RF of the WDR neurons, $\mathrm{MO}$ application produced a significant increase in their pinch/pressure RF but not in their tactile RF $(p<0.05$ and $p>0.8$, respectively) (Table 1 , Fig. 4 ). The two WDR neurons located in the superficial laminas, compared with the four WDR neurons in the deep laminas, had only a mild expansion of pinch/pressure RF $(<150 \%$ of control) after MO application. Compared with the $\mathrm{MSO} / \mathrm{MO}$ group, the additional glutamine pretreatment significantly restored the $\mathrm{MO}$-induced pinch/pressure and tactile $\mathrm{RF}$ expansion in both NS and WDR neurons in the $\mathrm{MSO}+\mathrm{Gln} / \mathrm{MO}$ group $(p<0.01-0.001)$ (Table 1$)$.

\section{Mechanical activation threshold}

In the PBS/MO group, the mechanical activation threshold of the six NS neurons significantly decreased from baseline to $54 \%$ of control after MO application $(p<0.05)$ (Table 1, Figs. 2, 5A). In the six WDR neurons, the threshold significantly decreased from baseline to $62 \%$ of control after MO application $(p<0.05)(\mathrm{Ta}-$ ble 1).

In the MSO/MO group, the activation threshold of the six NS neurons and the six WDR neurons was not significantly altered after MO application $(p>0.8)$, indicating that the MSO pretreatment had significantly attenuated the MO-induced decrease in threshold, compared with the PBS/MO group in the NS neurons $(p<0.001)$ (Table 1 , Fig. $5 A)$ and the six WDR neurons $(p<0.05)$ (Table 1$)$.

In the $\mathrm{MSO}+\mathrm{Gln} / \mathrm{MO}$ group, the activation threshold of the six NS neurons did not significantly change after MSO plus glutamine superfusion but significantly decreased to $70 \%$ of control after MO application $(p<0.05)$ (Fig. 2). The threshold of the six

activity
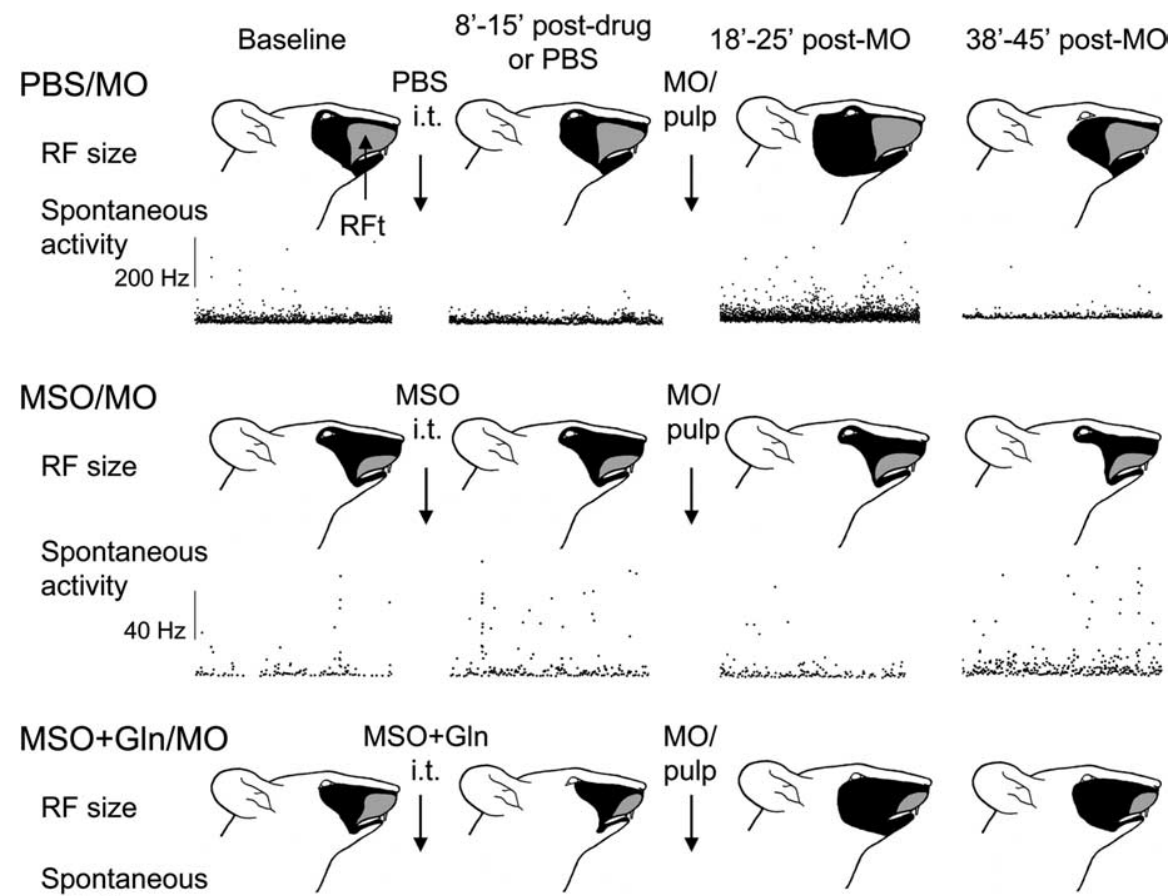

Figure 3. Examples showing M0-induced neuroplastic changes (central sensitization) in WDR neurons after continuous intrain an interspike instantaneous frequency distribution. The black area indicates the pinch/pressure RF. RFt refers to that part of the RF from which the neuron could be activated by tactile as well as pinch/pressure stimulation.

WDR neurons also significantly decreased (to $49 \%$ of control) after MO application. These data indicate that the additional glutamine pretreatment had restored the $\mathrm{MO}$-induced decrease in threshold in the MSO+Gln/MO group, compared with the MSO/MO group, in both NS neurons $(p<0.001)$ (Table 1, Figs. $2,5 A)$ and WDR neurons $(p<0.05)$ (Table 1$)$.

\section{Responses to graded pinch/pressure stimuli}

In the PBS/MO group, the six NS neurons comprising this group had a mean baseline response of $36.1 \pm 5.8$ spikes, which remained unchanged after PBS superfusion but significantly increased to $410 \%$ of control after MO application $(p<0.05)$ (Table 1, Figs. 2, 5B).

In the MSO/MO group, the six NS neurons had a mean baseline response of $89 \pm 28.7$ spikes, which was slightly decreased at the beginning of MSO superfusion but did not significantly increase after MO application $(p>0.8)$. Compared with the $\mathrm{PBS} / \mathrm{MO}$ group, the additional MSO pretreatment significantly attenuated the MO-induced increase in responses $(p<0.001)$ (Table 1, Figs. 2, 5B).

In the MSO $+\mathrm{Gln} / \mathrm{MO}$ group, the six NS neurons had a mean baseline response of $122.7 \pm 28.7$ spikes, which was only mildly increased after MSO plus glutamine superfusion but was markedly increased to $330 \%$ of control after MO application; however, this increase was not significant, probably because of the response variability of the individual neurons (Fig. 5B). Nonetheless, the differences in pinch/pressure-evoked responses after MO appli- 
A
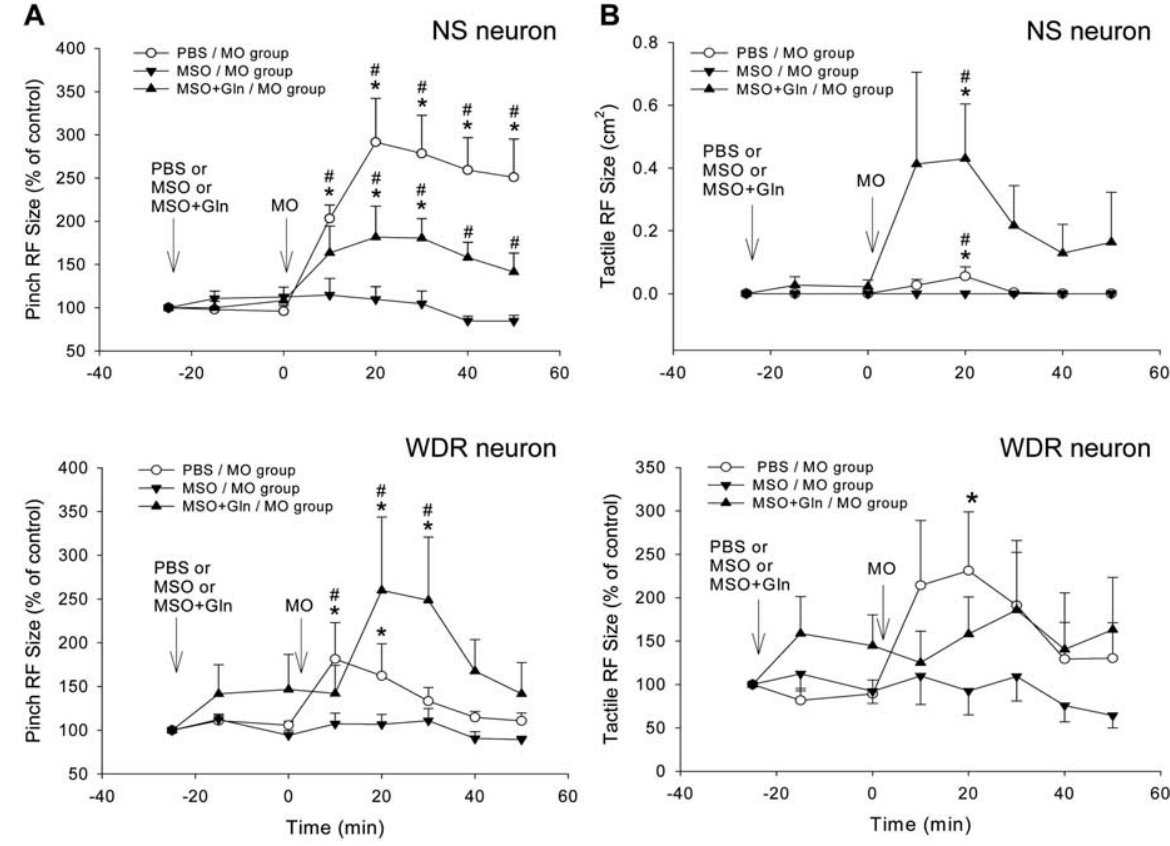

Figure 4. Changes in NS and WDR neuronal RF size induced by $M O$ application to the tooth pulp after continuous intrathecal superfusion of PBS, MSO, or MSO plus glutamine over MDH. Each group consists of six NS or six WDR neurons. A, Pinch/pressure RF. In the PBS/MO group, $\mathrm{M} 0$ application to the pulp produced significant increases in pinch/pressure RF size throughout the $50 \mathrm{~min}$ observation period ( $p<0.005$ and 0.001 for WDR and NS neurons, respectively, RM ANOVA; ${ }^{*} p<0.05$, Dunnett's test); in the $\mathrm{MSO} / \mathrm{MO}$ group, MSO superfusion strongly attenuated the M0-induced increases in pinch RF size ( $p>0.7$ and $p>0.2$ for NS and WDR neurons, respectively, RM ANOVA), whereas in the MSO $+G \ln / \mathrm{MO}$ group, the additional glutamine superfusion restored the M0-induced effect ( $p<0.005$ and $p<0.02$ for NS and WDR neurons, respectively, RM ANOVA). B, Tactile RF. In the PBS/MO group, M0 application to the pulp produced significant increases in tactile RF size of WDR neurons ( $p<0.05$, RM ANOVA on ranks; ${ }^{*} p<0.05$, Dunnett's test) and a transient appearance of novel tactile RF in some NS neurons ( $p<0.02$, RM ANOVA; ${ }^{*} p<0.05$, Dunnett's test); in the MSO/MO group, the MSO superfusion attenuated the MO-induced increases in tactile RF size of WDR neurons ( $p>0.4$, RM ANOVA) and prevented the appearance of novel tactile RF in NS neurons, whereas in the MSO $+G \ln / \mathrm{MO}$ group, the additional glutamine superfusion restored the M0 effects in NS but not in WDR neurons ( $p<0.005$ and $p>0.8$, respectively, RM ANOVA on ranks). There were significant differences between the PBS/MO and MSO/M0 groups and between $\mathrm{MSO}+\mathrm{Gln} / \mathrm{MO}$ and MSO/M0 groups in both NS and WDR neurons ( $p<0.01-0.001$, 2-way ANOVA) (also see Table 1) (" $p<$ 0.05 , Bonferroni $t$ test, which reflects a significance difference in values at the same post-M0 time point between two groups). Arrows indicate the application time (at $0 \mathrm{~min}$ ) of chemicals or PBS. Error bars represent SEM.

cation between the $\mathrm{MSO}+\mathrm{Gln} / \mathrm{MO}$ and $\mathrm{MSO} / \mathrm{MO}$ groups were significant, suggesting that glutamine pretreatment had restored the MO-induced increase in pinch/pressure-evoked responses in the $\mathrm{MSO}+\mathrm{Gln} / \mathrm{MO}$ group, compared with the $\mathrm{MSO} / \mathrm{MO}$ group $(p<0.02)$ (Figs. 2, 5B, Table 1).

\section{Spontaneous activity}

In the PBS/MO group (six NS, six WDR), only one NS neuron had low spontaneous activity $(<0.07 \mathrm{~Hz})$ at baseline; $\mathrm{MO}$ application to the tooth pulp increased its activity to $25 \mathrm{~Hz}$. MO also produced a transient weak response in two other NS neurons. All six WDR neurons had spontaneous activity $(0.01-14 \mathrm{~Hz})$; $\mathrm{MO}$ application increased this activity to a higher level $(0.02-29.7 \mathrm{~Hz})$. However, the changes in firing rates after MO application in either group of NS or WDR neurons were not significant.

In the MSO/MO group (six NS, six WDR), only one NS neuron had baseline spontaneous activity $(1.3 \mathrm{~Hz})$, and it increased to 4.4 and $4.7 \mathrm{~Hz}$ after MSO superfusion and MO application, respectively. All six WDR neurons had baseline spontaneous activity $(0.01-0.5 \mathrm{~Hz})$ that was unchanged in all but one after both MSO and MO application. In either NS or WDR neurons, there was no significant difference between the baseline and $18 \mathrm{~min}$ post-MO activities. In comparisons between groups, there was no significant difference between the $\mathrm{MSO} / \mathrm{MO}$ and $\mathrm{PBS} / \mathrm{MO}$ groups in the NS neuronal activity, but the spontaneous activity of the WDR neurons was significantly lower in the $\mathrm{MSO} / \mathrm{MO}$ group than in the PBS/MO group $(p<$ $\left.0.001 ; F_{(1,80)}=14.8\right)$.

In the $\mathrm{MSO}+\mathrm{Gln} / \mathrm{MO}$ group (six NS, six WDR), none of the six NS neurons had spontaneous activity at baseline. MO application produced a low level of activity $(0.02-0.1 \mathrm{~Hz})$ in two neurons. All six WDR neurons had low baseline spontaneous activity $(<0.9 \mathrm{~Hz})$. MO application produced an increase in activity in all six neurons; interestingly, the spontaneous activity of the four neurons located in the deep laminas increased to a much higher level (up to $17 \mathrm{~Hz}$ ) than that of the two neurons in the superficial laminas $(0.2-0.3 \mathrm{~Hz})$. However, probably because of the variability between neurons in spontaneous activity, the differences between the baseline and 18 min post-MO activities were not significant. In comparisons between groups, there was no significant difference between the $\mathrm{MSO}+\mathrm{Gln} / \mathrm{MO}$ and $\mathrm{MSO} / \mathrm{MO}$ groups for the WDR neurons, but the spontaneous activity of the NS neurons was significantly different between the two groups ( $p<$ $\left.0.003 ; F_{(1,80)}=5.1\right)$. Examples of spontaneous activity of WDR neurons from the above three groups are shown in Figure 3.

\section{Effects of MSO or glutamine}

In experiments in which six NS neurons were tested with either MSO alone or glutamine alone or glutamine followed by $\mathrm{MO}$ application, there were no significant changes in spontaneous activity, RF size, mechanical activation threshold, or responses to graded stimuli during the $50 \mathrm{~min}$ of superfusion of MSO or glutamine alone (all $p>0.2$ ), and glutamine followed by $\mathrm{MO}$ application did not interfere with the MO-induced central sensitization.

\section{Discussion}

The present study is the first documentation that continuous intrathecal superfusion of an inhibitor (MSO) of GS activity in astroglia strongly attenuates central sensitization induced in functionally identified nociceptive (WDR and NS) neurons in V subnucleus caudalis (also termed MDH) (Dubner and Bennett, 1983; Sessle, 2000). Furthermore, this study has documented that the MSO-mediated reduction in the MDH central sensitization induced by application of the inflammatory irritant MO to the tooth pulp can be restored by simultaneous intrathecal superfusion of glutamine, which is a key component of the glutamateglutamine shuttle. No such effects were seen when PBS superfusion (as control) in MDH preceded the MO application to the pulp, consistent with our previous several findings that MO application to the pulp produces MDH central sensitization reflected in increases in spontaneous activity, RF size, and responses to noxious stimuli and a decrease in mechanical activation threshold induced by MO application to orofacial tis- 

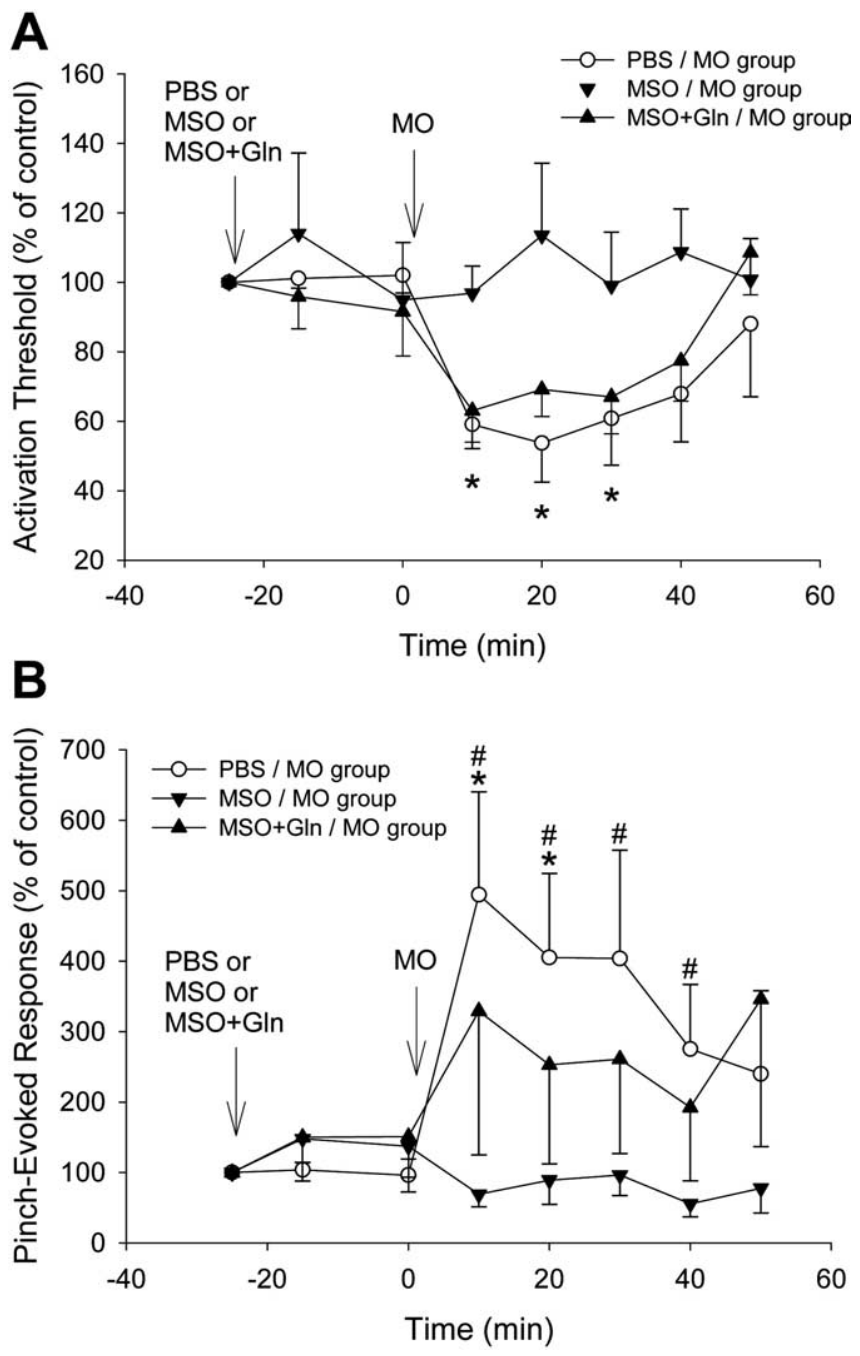

Figure 5. Changes in mechanical activation threshold and responses to graded mechanical stimuli of NS neurons induced by $\mathrm{M} 0$ application to the tooth pulp after continuous intrathecal superfusion of PBS, MSO, or MSO + glutamine over MDH. Each group consists of six NS neurons. $A$, In the PBS/M0 group, M0 application to the pulp produced significant decreases in threshold during the 50 min observation period ( $p<0.001$, RM ANOVA; ${ }^{*} p<0.05$, Dunnett's test); in the MSO/M0 group, M0 application no longer produced decreases in threshold ( $p>0.8, \mathrm{RM}$ ANOVA); in the $\mathrm{MSO}+\mathrm{Gln} / \mathrm{MO}$ group, the additional glutamine superfusion restored the M0induced decreases in threshold ( $p<0.01$, RM ANOVA). There were significant differences between the PBS/MO or MSO + GIn/MO and MSO/M0 groups (both $p<0.001$, 2-way ANOVA). $B$, In the PBS/M0 group, $\mathrm{MO}$ application to the pulp produced significant increases in responses during the 50 min observation period ( $p<0.002$, RM ANOVA; ${ }^{*} p<0.05$, Dunnett's test), whereas in the MSO/M0 group, the MSO superfusion strongly attenuated the M0-induced increases in responses ( $p>0.4, \mathrm{RM}$ ANOVA). In the MSO + Gln/M0 group, the additional glutamine superfusion moderately reversed this attenuation. There were significant differences between the PBS/M0 or MSO $+\mathrm{Gln} / \mathrm{M} 0$ and $\mathrm{MSO} / \mathrm{M} 0$ groups $(p<0.001$ or $p<0.02$, respectively, 2-way ANOVA; ${ }^{\#} p<0.05$, Bonferroni $t$ test). Arrows indicate the application time (at 0 $\mathrm{min}$ ) of chemicals or PBS. Error bars represent SEM.

sues, including the tooth pulp (Hu et al., 1992; Yu et al., 1993; Chiang et al., 1998, 2005; Xie et al., 2007).

The present findings are also consistent with previous behavioral and immunohistochemical studies (Watkins et al., 1997, 2001; Yeo et al., 2001; Piao et al., 2006; Lan et al., 2007), which demonstrated that astroglia and microglia are involved in the hyperalgesia induced by formalin application to the rat's hindpaw that can be attenuated by intrathecal administration of an astroglial metabolic inhibitor (FA or fluorocitrate) (Watkins et al., 1997; Lan et al., 2007). We have also recently shown that application of FA blocks central sensitization induced by the application of $\mathrm{MO}$ to the pulp in functionally identified nociceptive neurons in the MDH (Xie et al., 2007). Furthermore, upregulation of astroglial markers examined by immunostaining and reverse transcription-PCR assays has also been observed at the subacute $(4 \mathrm{~h})$ and chronic ( $4-14 \mathrm{~d})$ phases of inflammation induced by intraplantar injection of complete Freund's adjuvant (Raghavendra et al., 2004), and similar findings were observed after trigeminal sensory nerve injury (Piao et al., 2006); however, in other studies, immunohistochemical data indicative of spinal astroglia activation were detected earlier than microglia activation and occurred as early as $0.5-1 \mathrm{~h}$ after subcutaneous formalin injection (Sweitzer et al., 1999; Qin et al., 2006).

Astroglia provide an array of supportive, nutritional, developmental, and homeostatic functions to surrounding neurons and can also modulate synaptic transmission (Allen and Barres, 2005; Fitzgerald, 2005; Araque, 2006; Haydon and Carmignoto, 2006). A key and specific process of astroglial function is the glutamateglutamine shuttle, which includes the uptake of the excess extrasynaptic glutamate and the production (via astroglial enzyme GS) and release from astroglia of glutamine, which is then taken up by neuronal elements to replenish the supply of glutamate (Zwingmann and Leibfritz, 2003; Hertz, 2004; Hertz and Zielke, 2004; Fonseca et al., 2005), which plays a major role in central sensitization (Dubner and Basbaum, 1994; Sessle, 2000; Woolf and Salter, 2006). In the spinal cord as well as the brain, GS exists in astroglia and oligodendrocytes but not in neurons (Norenberg, 1979; Warringa et al., 1988; Cammer, 1990; Suarez et al., 1997, 2002); however, some earlier radio-assay studies reported that GS may also exist in cultured cortical neurons (Zwingmann and Leibfritz, 2003). Although there is a lack of direct evidence for altered astroglial GS activity in the spinal cord related to peripheral inflammatory events, recent studies have reported that GS activity is increased in the white and gray matters of lumbar as well as cervical enlargements $7 \mathrm{~d}$ after spinal cord injury in rats (Benton et al., 2000). Furthermore, the injection of the inflammatory irritant capsaicin into the dorsal rump region significantly increased the glial fibrillary acidic protein, colocalized with GS immunoreactivity in the arcuate-median eminence of hypothalamus (Okere and Waterhouse, 2004).

MSO has been widely used as a GS inhibitor in astroglia in studies of hyperammonemia, memory consolidation, and seizures, and its effects can be counteracted by glutamine application (Bacci et al., 2002; Suarez et al., 2002; Gibbs and Hertz, 2005; Tanigami et al., 2005; Liang et al., 2006). One recent study has used MSO to examine the effect of inflammatory stimuli on activation of GS in astroglia and found that GS activation is related to glutamatergic receptor activation (Muscoli et al., 2005). Because GS exists in the MDH (Norenberg, 1979; Cammer, 1990), the superfusion of MSO onto the brainstem overlying $\mathrm{MDH}$ as perfused in the present study would directly target the GS activity in $\mathrm{MDH}$ astroglia, and so the suppressive effect of $\mathrm{MSO}$ on $\mathrm{MDH}$ central sensitization could be attributable to its action on astroglial GS function in the MDH. However, we cannot be certain of the exact site of action of the superfused chemicals, and the use of other techniques (e.g., microdialysis) will be needed to clarify the exact site of action. Furthermore, because MSO (100-300 $\mu \mathrm{M})$ application to cortical slices induces glutamate and glutamine release and a sustained cortical neuronal depolarization, which could be partially blocked by an NMDA antagonist (Albrecht and Norenberg, 1990; Shaw et al., 1999; Zielinska et al., 2004), it is possible that the MSO effects we have documented in the $\mathrm{MDH}$ were caused by a direct action of MSO on MDH neuronal excit- 
ability. However, the present study used only a low dose of MSO $(100 \mu \mathrm{M})$, and there were no significant changes in nociceptive neuronal properties during its superfusion alone, except for a mild but nonsignificant increase in spontaneous activity or neuronal responses to noxious stimuli in only three neurons. The lack of any observed significant effects of MSO alone, in contrast to our findings that it significantly attenuates $\mathrm{MO}$-induced central sensitization, suggests that its action on astroglial GS is not evident in basal conditions but is apparent in hyperexcitable states, consistent with findings by ourselves and others that glia may not affect basal nociceptive processing but rather participate in exaggerated pain states (Watkins and Maier, 2005; Xie et al., 2007). It is also noteworthy that an excess of glutamine in the CNS is involved in ammonia neurotoxicity (e.g., hyperammonemia and hepatic encephalopathy), possibly through its detrimental effects on mitochondrial function (Albrecht and Norenberg, 2006). The dose of glutamine superfusion $(250 \mu \mathrm{M})$ used in the present study was also selected at a level below the rat's cerebral basal interstitial concentration $(385 \mu \mathrm{M})$ (Kanamori and Ross, 2004) to avoid neuronal excitation elicited by too high a dose of glutamine, and control experiments with superfusion of glutamine also resulted in no significant changes in $\mathrm{MDH}$ neuronal properties.

Thus, given this and the earlier findings of MSO effects on astroglial GS, we interpret our findings as indicative of the MSO inhibition of astroglial GS and thereby modulation of the glutamate-glutamine shuttle. Such a view is further supported by our recent documentation that continuous intrathecal superfusion over $\mathrm{MDH}$ of FA can also attenuate central sensitization in $\mathrm{MDH}$ nociceptive neurons induced by $\mathrm{MO}$ application to the tooth pulp (Xie et al., 2007). Whereas FA is a specific inhibitor of the metabolic enzyme aconitase in the astroglial tricarboxylic acid cycle (Fonnum et al., 1997; Schousboe and Waagepetersen, 2006), MSO affects the downstream consequences of astroglial function by inhibiting the production and release of glutamine from astroglia, which is essential for glutamatergic neurotransmission in pathological pain states. Our FA and MSO studies collectively represent the first documentation that the functional integrity and metabolic activity of astroglia are essential for the initiation of central sensitization in functionally identified dorsal horn nociceptive neurons. Furthermore, these data as well as our recent findings that MSO intrathecal administration can also reduce the central sensitization induced by chronic tooth pulp inflammation and inferior alveolar nerve transection ( $\mathrm{Hu}$ et al., 2006; Suzuki et al., 2007) indicate that astroglia may be intimately involved in the maintenance as well as development of central sensitization induced by inflammation and nerve injury.

\section{References}

Albrecht J, Norenberg MD (1990) L-methionine-DL-sulfoximine induces massive efflux of glutamine from cortical astrocytes in primary culture. Eur J Pharmacol 182:587-589.

Albrecht J, Norenberg MD (2006) Glutamine: a Trojan horse in ammonia neurotoxicity. Hepatology 44:788-794.

Allen NJ, Barres BA (2005) Signaling between glia and neurons: focus on synaptic plasticity. Curr Opin Neurobiol 15:542-548.

Araque A (2006) Astrocyte-neuron signaling in the brain-implications for disease. Curr Opin Investig Drugs 7:619-624.

Bacci A, Sancini G, Verderio C, Armano S, Pravettoni E, Fesce R, Franceschetti S, Matteoli M (2002) Block of glutamate-glutamine cycle between astrocytes and neurons inhibits epileptiform activity in hippocampus. J Neurophysiol 88:2302-2310.

Benton RL, Ross CD, Miller KE (2000) Glutamine synthetase activities in spinal white and gray matter 7 days following spinal cord injury in rats. Neurosci Lett 291:1-4.
Blin M, Crusio WE, Hevor T, Cloix J-F (2002) Chronic inhibition of glutamine synthetase is not associated with impairment of learning and memory in mice. Brain Res Bull 57:11-15.

Cammer W (1990) Glutamine synthetase in the central nervous system is not confined to astrocytes. J Neuroimmunol 26:173-178.

Chiang CY, Park SJ, Kwan CL, Hu JW, Sessle BJ (1998) NMDA receptor mechanisms contribute to neuroplasticity induced in caudalis nociceptive neurons by tooth pulp stimulation. J Neurophysiol 80:2621-2631.

Chiang CY, Zhang S, Xie YF, Hu JW, Dostrovsky JO, Sessle BJ (2005) Endogenous ATP involvement in mustard oil-induced central sensitization in trigeminal subnucleus caudalis (medullary dorsal horn). J Neurophysiol 94:1751-1760.

Chiang CY, Wang J, Xie YF, Zhang S, Hu JW, Dostrovsky JO, Sessle BJ (2006) Modulation of glutamine synthetase (GS) activity in astroglia affects central sensitization in trigeminal subnucleus caudalis (medullary dorsal horn). Soc Neurosci Abstr 32:535.2.

Dubner R, Basbaum AI (1994) Spinal dorsal horn plasticity following tissue or nerve injury. In: Textbook of pain, Ed 3 (Wall PD, Melzack R, eds), pp 225-241. New York: Churchill Livingstone.

Dubner R, Bennett GJ (1983) Spinal and trigeminal mechanisms of nociception. Annu Rev Neurosci 6:381-418.

Fitzgerald M (2005) The development of nociceptive circuits. Nat Rev Neurosci 6:507-520.

Fonnum F, Johnsen A, Hassel B (1997) Use of fluorocitrate and fluoroacetate in the study of brain metabolism. Glia 21:106-113.

Fonseca LL, Monteiro MA, Alves PM, Carrondo MJ, Santos H (2005) Cultures of rat astrocytes challenged with a steady supply of glutamate: new model to study flux distribution in the glutamate-glutamine cycle. Glia 51:286-296.

Frank MG, Baratta MV, Sprunger DB, Watkins LR, Maier SF (2007) Microglia serve as a neuroimmune substrate for stress-induced potentiation of CNS pro-inflammatory cytokine responses. Brain Behav Immun 21:47-59.

Gibbs ME, Hertz L (2005) Importance of glutamate-generating metabolic pathways for memory consolidation in chicks. J Neurosci Res 81:293-300.

Haydon PG, Carmignoto G (2006) Astrocyte control of synaptic transmission and neurovascular coupling. Physiol Rev 86:1009-1031.

Hertz L (2004) Intercellular metabolic compartmentation in the brain: past, present and future. Neurochem Int 45:285-296.

Hertz L, Zielke HR (2004) Astrocytic control of glutamatergic activity: astrocytes as stars of the show. Trends Neurosci 27:735-743.

Hu B, Chiang CY, Hu JW, Dostrovsky JO, Sessle BJ (2002) P2X receptors in trigeminal subnucleus caudalis modulate central sensitization in trigeminal subnucleus oralis. J Neurophysiol 88:1614-1624.

Hu JW, Sessle BJ, Raboisson P, Dallel R, Woda A (1992) Stimulation of craniofacial muscle afferents induces prolonged facilitatory effects in trigeminal nociceptive brain-stem neurones. Pain 48:53-60.

Hu JW, Tsuboi Y, Sessle BJ, Iwata K (2006) Modulation of glutamine synthetase (GS) activity in astroglia affects nociceptive behaviour in rats with chronic pulp exposure. Soc Neurosci Abstr 32:50.4.

Inoue K, Tsuda M, Koizumi S (2004) Chronic pain and microglia: the role of ATP. Novartis Found Symp 261:55-64.

Kanamori K, Ross BD (2004) Quantitative determination of extracellular glutamine concentration in rat brain, and its elevation in vivo by system $\mathrm{A}$ transport inhibitor, alpha-(methylamino)isobutyrate. J Neurochem 90:203-210.

Lan L, Yuan H, Duan L, Cao R, Gao B, Shen J, Xiong Y, Chen LW, Rao ZR (2007) Blocking the glial function suppresses subcutaneous formalininduced nociceptive behavior in the rat. Neurosci Res 57:112-119.

Liang SL, Carlson GC, Coulter DA (2006) Dynamic regulation of synaptic GABA release by the glutamate-glutamine cycle in hippocampal area CA1. J Neurosci 26:8537-8548.

Miller G (2005) The dark side of glia. Science 308:778-781.

Muscoli C, Visalli V, Colica C, Nistico R, Palma E, Costa N, Rotiroti D, Nistico G, Mollace V (2005) The effect of inflammatory stimuli on NMDA-related activation of glutamine synthase in human cultured astroglial cells. Neurosci Lett 373:184-188.

Norenberg MD (1979) Distribution of glutamine synthetase in the rat central nervous system. J Histochem Cytochem 27:756-762.

Okere CO, Waterhouse BD (2004) Capsaicin increases GFAP and glutamine synthetase immunoreactivity in rat arcuate nucleus and median eminence. NeuroReport 15:255-258. 
Park SJ, Chiang CY, Hu JW, Sessle BJ (2001) Neuroplasticity induced by tooth pulp stimulation of nociceptive neurons in trigeminal subnucleus oralis involves NMDA receptor mechanisms. J Neurophysiol 85:1836-1846.

Piao ZG, Cho IH, Park CK, Hong JP, Choi SY, Lee SJ, Lee S, Park K, Kim JS, Oh SB (2006) Activation of glia and microglial p38 MAPK in medullary dorsal horn contributes to tactile hypersensitivity following trigeminal sensory nerve injury. Pain 121:219-231.

Qin M, Wang JJ, Cao R, Zhang H, Duan L, Gao B, Xiong YF, Chen LW, Rao ZR (2006) The lumbar spinal cord glial cells actively modulate subcutaneous formalin induced hyperalgesia in the rat. Neurosci Res 55:442-450.

Raghavendra V, Tanga FY, DeLeo JA (2004) Complete Freunds adjuvantinduced peripheral inflammation evokes glial activation and proinflammatory cytokine expression in the CNS. Eur J Neurosci 20:467-473.

Raivich G (2005) Like cops on the beat: the active role of resting microglia. Trends Neurosci 28:571-573.

Schousboe A, Waagepetersen HS (2006) Glial modulation of GABAergic and glutamatergic neurotransmission. Curr Top Med Chem 6:929-934.

Sessle BJ (2000) Acute and chronic craniofacial pain: brainstem mechanisms of nociceptive transmission and neuroplasticity, and their clinical correlates. Crit Rev Oral Biol Med 11:57-91.

Shaw CA, Bains JS (1998) Did consumption of flour bleached by the agene process contribute to the incidence of neurological disease? Med Hypotheses 51:477-481.

Shaw CA, Bains JS, Pasqualotto BA, Curry K (1999) Methionine sulfoximine shows excitotoxic actions in rat cortical slices. Can J Physiol Pharmacol 77:871-877.

Shin DW, Yoon YS, Matsumoto M, Huang W, Ceraulo P, Young W (2003) Glutamine synthetase induced spinal seizures in rats. Yonsei Med J 44:125-132.

Suarez I, Bodega G, Arilla E, Fernandez B (1997) Region-selective glutamine synthetase expression in the rat central nervous system following portocaval anastomosis. Neuropathol Appl Neurobiol 23:254-261.

Suarez I, Bodega G, Fernandez B (2002) Glutamine synthetase in brain: effect of ammonia. Neurochem Int 41:123-142.

Suzuki I, Ogawa A, Sessle BJ, Iwata K (2007) Effect of astroglial inhibitor on trigeminal subnucleus caudalis neurons in rats with inferior alveolar nerve transaction (in Japanese). Jpn Assoc Study Pain Abstr 22:97.

Sweitzer SM, Colburn RW, Rutkowski M, DeLeo JA (1999) Acute peripheral inflammation induces moderate glial activation and spinal IL-1beta expression that correlates with pain behavior in the rat. Brain Res 829:209-221.

Tanga FY, Raghavendra V, DeLeo JA (2004) Quantitative real-time RTPCR assessment of spinal microglial and astrocytic activation markers in a rat model of neuropathic pain. Neurochem Int 45:397-407.
Tanigami H, Rebel A, Martin LJ, Chen TY, Brusilow SW, Traystman RJ, Koehler RC (2005) Effect of glutamine synthetase inhibition on astrocyte swelling and altered astroglial protein expression during hyperammonemia in rats. Neuroscience 131:437-449.

Tsuda M, Shigemoto-Mogami Y, Koizumi S, Mizokoshi A, Kohsaka S, Salter MW, Inoue K (2003) P2X4 receptors induced in spinal microglia gate tactile allodynia after nerve injury. Nature 424:778-783.

Tsuda M, Inoue K, Salter MW (2005) Neuropathic pain and spinal microglia: a big problem from molecules in "small" glia. Trends Neurosci 28:101-107.

Warringa RAJ, van Berlo MF, Klein W, Lopes-Cardozo M (1988) Cellular location of glutamine synthetase and lactate dehydrogenase in oligodendrocyte-enriched cultures from rat brain. J Neurochem 50:1461-1468.

Watkins LR, Maier SF (2005) Immune regulation of central nervous system functions: from sickness responses to pathological pain. J Intern Med 257:139-155.

Watkins LR, Martin D, Ulrich P, Tracey KJ, Maier SF (1997) Evidence for the involvement of spinal cord glia in subcutaneous formalin induced hyperalgesia in the rat. Pain 71:225-235.

Watkins LR, Milligan ED, Maier SF (2001) Glial activation: a driving force for pathological pain. Trends Neurosci 24:450-455.

Woolf CJ, Salter MW (2006) Plasticity and pain: role of the dorsal horn. In: Wall and Melzack's textbook of pain, Chap 5, Ed 5 (McMahon S, Koltzenburg M, eds), pp 91-105. London: Elsevier.

Xie YF, Zhang S, Chiang CY, Hu JW, Dostrovsky JO, Sessle BJ (2007) Involvement of glia in central sensitization in trigeminal subnucleus caudalis (medullary dorsal horn). Brain Behav Immun 21:634-641.

Yeo JF, Liu HP, Leong SK (2001) Sustained microglial immunoreactivity in the caudal spinal trigeminal nucleus after formalin injection. J Dent Res 80:1524-1529.

Yu X-M, Sessle BJ, Hu JW (1993) Differential effects of cutaneous and deep application of inflammatory irritant on mechanoreceptive field properties of trigeminal brain stem nociceptive neurons. J Neurophysiol 70:1704-1707.

Zhang FY, Wan Y, Zhang ZK, Light AR, Fu KY (2007) Peripheral formalin injection induces long-lasting increases in cyclooxygenase 1 expression by microglia in the spinal cord. J Pain 8:110-117.

Zielinska M, Stafiej A, Law RO, Albrecht J (2004) Effects of methionine sulfoximine on the glutamine and glutamate content and cell volume in rat cerebral cortical slices: involvement of mechanisms not related to inhibition of glutamine synthesis. Neurotoxicology 25:443-449.

Zwingmann C, Leibfritz D (2003) Regulation of glial metabolism studied by ${ }^{13}$ C-NMR. NMR Biomed 16:370-399. 